\title{
Unveiling Covariate Inclusion Structures In Economic Growth Regressions Using Latent Class Analysis*
}

\author{
Jesus Crespo Cuaresma $a^{\mathrm{a}, \mathrm{b}, \mathrm{c}, \mathrm{d}}$, Bettina Grün ${ }^{\mathrm{e}}$, Paul Hofmarcher ${ }^{\mathrm{a}}$, Stefan \\ Humer $^{\mathrm{a}}$, and Mathias Moser ${ }^{\mathrm{a}}$ \\ ${ }^{\mathrm{a}}$ Department of Economics, Vienna University of Economics and Business (WU) \\ ${ }^{\mathrm{b}}$ Austrian Institute of Economic Research (WIFO) \\ ${ }^{\mathrm{c}}$ International Institute of Applied System Analysis (IIASA) \\ $\mathrm{d}_{\text {Wittgenstein Centre for Demography and Global Human Capital (WIC) }}$ \\ ${ }^{\mathrm{e}}$ Department of Applied Statistics, Johannes Kepler University Linz (JKU)
}

\begin{abstract}
We propose the use of Latent Class Analysis methods to analyze the covariate inclusion patterns across specifications resulting from Bayesian Model Averaging exercises. Using Dirichlet Process clustering, we are able to identify and describe dependency structures among variables in terms of inclusion in the specifications that compose the model space. We apply the method to two datasets of potential determinants of economic growth. Clustering the posterior covariate inclusion structure of the model space formed by linear regression models reveals interesting patterns of complementarity and substitutability across economic growth determinants.
\end{abstract}

JEL Classification: C11, C21, O47.

Keywords: Economic Growth Determinants, Bayesian Model Averaging, Latent Class Analysis, Dirichlet Processes.

\footnotetext{
${ }^{*}$ The authors would like to thank Sylvia Frühwirth-Schnatter, two anonymous referees and the participants at the Second Bayesian Young Statisticians Meeting for helpful comments. Corresponding Author: Jesus Crespo Cuaresma. Address: Welthandelsplatz 1, 1020 Vienna, Austria, Tel: +43(0)131336-4530, Fax: +43(0)131336-728, Email: jcrespo@wu.ac.at. Paul Hofmarcher's research is supported by funds of the Oesterreichische Nationalbank (Oesterreichische Nationalbank, Anniversary Fund, project number: 14663).
} 


\section{Introduction}

Bayesian Model Averaging (BMA) has become a popular tool for economic growth applications in economics (for a comprehensive introduction to BMA, see Hoeting et al., 1999). The so-called open-endedness of economic theory concerning the factors driving income per capita differences across countries (Brock and Durlauf, 2001) allows the empirical researcher to specify a large number of models to quantify the effect of potential drivers on economic growth. The use of techniques that explicitly assess model uncertainty (mostly within the class of linear regression models) has thus become widespread in econometric research dealing with the empirical determinants of income growth differences across countries (for some seminal contributions to this literature, see e.g. Fernandez et al., 2001; Sala-i Martin et al., 2004; Masanjala and Papageorgiou, 2008; Durlauf et al., 2008; Ley and Steel, 2009b).

Economic growth applications of BMA tend to quantify the relative importance of a given covariate by calculating its so-called posterior inclusion probability (PIP), which is defined as the sum of posterior probabilities of specifications which contain that particular variable. Such a statistic has become a standard tool in econometric applications of BMA and is routinely used to measure the relative importance of different potential drivers of income growth differences across economies. While standard PIPs are intuitive measures that provide valuable insights into the overall importance of individual covariates as economic growth determinants, they face a number of shortcomings. The PIP neglects the heterogeneity across typical model specifications and accordingly does not inform about whether the degree of importance of the variable is evenly spread across potential specifications (that is, it is relatively independent of whether other covariates are part of the model) or, on the contrary, it is particular to specific combinations of explanatory variables.

Previous work assessing joint covariate inclusion in BMA applications has focused on capturing relevant dependency structures using bivariate measures, that is, concentrating on the analysis of the joint posterior distribution of the inclusion of pairs of variables over the model space. Such a concept has been quantified in the form of bivariate jointness measures in the context of BMA, put forward first by G. Doppelhofer and M. Weeks in a working paper of 2005 (Doppelhofer et al., 2005), which got published in a slightly different version as Doppelhofer and Weeks (2009a). Ley and Steel (2007), Strachan (2009) and Ley and Steel (2009a) offer alternative measures of jointness. In particular, Ley and Steel (2007) formulate a set of properties for jointness measures and show that Doppelhofer and Weeks's statistics do not fulfill them. Additionally, they propose two other indices which satisfy all of their suggested properties. Strachan (2009) shows that the interpretability of the jointness measure of Doppelhofer and Weeks (2009a) may be limited in contexts where one or both of the analyzed variables have a negligible PIP and offers yet another measure in order to tackle this shortcoming. Doppelhofer and Weeks (2009b), on the other hand, argue that another desirable property of jointness measures happens to be fulfilled by their indicator but not accounted for in the indices of Ley and Steel (2007) or Strachan (2009). ${ }^{1}$

\footnotetext{
${ }^{1}$ Interestingly, the measures proposed by Doppelhofer and Weeks (2009a), Ley and Steel (2007) and Strachan
} 
In this paper we propose an alternative approach aimed at succinctly and comprehensibly describing the dependency structure across variables in the model space using latent class analysis (LCA, see, e.g., Vermunt and Magidson, 2002) and apply it to economic growth regressions. This method was first introduced by Lazarsfeld (1950) to describe dependency structures between observed discrete variables based on latent traits and has gained widespread popularity in such research fields as psychometrics or political science (see, e.g., Breen, 2000; Blaydes and Linzer, 2008). The main idea behind LCA is to relate the realizations of observed variables to an unobserved, categorical latent variable which captures the dependency structure between the observed variables. This latent variable groups observations in such a way that the dependency between variables is reduced to a minimum within groups. By applying LCA methods to the covariate inclusion structure of best models identified by BMA, we are able to capture the dependency patterns across included covariates through a (unobserved) latent variable which induces classes with independent covariates conditional on class membership. Such a setting implies that PIPs within clusters constitute sufficient information to describe the importance of the variables and the differences of PIPs between clusters are representative of the dependencies in the inclusion of a covariate with respect to (all) other variables.

The method proposed in this paper provides a tool for applied econometricians that goes beyond the identification of individual robust determinants of socioeconomic variables by distilling the joint covariate structures that underlie the distribution of the posterior model probability across specifications. Suitable theoretical frameworks based on the results of the clustering can then be inferred based on the identity of the corresponding groups of variables. In the spirit of Durlauf et al. (2008), the applied researcher may be interested in incorporating prior beliefs about the relative importance of some theoretical frameworks (defined over the joint prior inclusion probability of certain covariate groups) in order to assess the evidence for or against them. The modeling tool provided by our method is able to incorporate this information in a straightforward manner.

We apply this approach to the two datasets that have been most widely used for assessing the robustness of economic growth determinants (those in Fernandez et al., 2001, and Sala-i Martin et al., 2004, henceforth FLS and SDM, respectively). Our results for the FLS dataset reveal patterns of complementarity and substitutability across geographical, institutional and religious variables. For the SDM dataset, we find that the importance of the variable related to malaria prevalence is highly dependent on the inclusion of other covariates in the specification. The insights gained from the clustering exercise for the SDM dataset partly reconcile some of the contradictory results found in the literature concerning the importance of malaria prevalence as a determinant of income growth differences across countries (see for example Sala-i Martin et al., 2004; Schneider and Wagner, 2012; Hofmarcher et al., 2014).

The remainder of this paper is structured as follows. In Section 2, we present the econometric setting used to analyze the anatomy of covariate inclusion over the model space within BMA

(2009) were independently developed earlier in the context of data mining. The statistic of Doppelhofer et al. (2005) is known as log-ratio, the measures of Ley and Steel (2007) are related to the Jaccard index. The index of Doppelhofer and Weeks (2009a) is known as odds-ratio and Strachan (2009)'s measure is closely related to the so-called two-way support (see Tan et al., 2004; Glass, 2013). 
applications and outline the LCA approach. Section 3 presents the results of the LCA analysis applied to the set of best models identified for the FLS and SDM datasets. Section 4 concludes and proposes further paths of research.

\section{Evaluating Covariate Inclusion Dependency Using Latent Class Analysis}

\subsection{Model Uncertainty and Economic Growth Determinants: The Econometric Framework}

The standard setting for BMA analysis in the framework of cross-country growth regressions assumes that the growth rate of income per capita $(y)$ can be linearly related to a group of covariates $\left(X_{j}\right)$ chosen from a set of potential growth determinants $(X)$. Assuming that $n$ observations are available, a typical linear regression model $\left(M_{j}\right)$ is given by

$$
y \mid \alpha, \beta_{j}, \sigma \sim N\left(\alpha \iota+X_{j} \beta_{j}, \sigma^{2} I\right)
$$

where $\iota$ is a column vector of ones of dimension $n$. Assuming that a total of $K$ variables are available, inference on a quantity of interest $(\Delta)$ is given by

$$
p(\Delta \mid y)=\sum_{j=1}^{2^{K}} p\left(\Delta \mid y, M_{j}\right) p\left(M_{j} \mid y\right),
$$

where $p\left(M_{j} \mid y\right)$ is the posterior model probability, which in turn is proportional to the product of the prior model probability $p\left(M_{j}\right)$ and its marginal likelihood $p\left(y \mid M_{j}\right)$. After eliciting priors over model-specific parameters $\left(p\left(\beta_{j} \mid M_{j}\right)\right.$ and $\left.p\left(\sigma \mid M_{j}\right)\right)$, as well as over models $\left(p\left(M_{j}\right)\right)$, posterior model probabilities and thus the posterior distributions given by equation (2) can be computed. The problems caused by the exorbitantly large number of summands in equation (2) when $K$ is not small can be overcome in a straightforward manner by sampling from the model space using Markov Chain Monte Carlo (MCMC) methods (Madigan and York, 1995).

In the spirit of the literature on jointness in BMA applications, we propose to analyze the anatomy of the set of models sampled by the Markov chain in order to carry out inference about the covariate inclusion structures existing in the model space. While existing jointness measures tend to concentrate on the analysis of the $K \times K$ matrix of bivariate inclusion frequencies in the Markov chain, we aim at gaining knowledge about the overall structure of covariate inclusion by analyzing the full $M \times K$ matrix of inclusion profiles of the specifications sampled by the Markov chain, where $M$ is the number of sampled models. A model profile $\gamma_{i}$, for $i=1, \ldots, M$ (that is, one of the rows of the matrix), is a $K$-dimensional vector of ones and zeros indicating the variables which are included in model $i$, with typical element $\gamma_{i k}=1$ if variable $k$ is part of model $i$ and $\gamma_{i k}=0$ otherwise. We propose to perform the analysis of the inclusion patterns over the model space assuming the existence of implicit latent groups to which model specifications are assigned 
depending on their covariate inclusion pattern.

\subsection{Latent Classes and Covariate Inclusion: A Bayesian Approach Using Dirichlet Processes}

We propose to use a method that resembles existing BMA applications dealing with the computation of jointness measures among covariates. It takes a two-step approach in terms of analyzing the posterior probability distribution over model specifications obtained using standard BMA methods. Using clustering methods based on LCA, it aims at unveiling clusters of model profiles among the specifications sampled in the Markov chain Monte Carlo model composite procedure.

Following the methods put forward by Molitor et al. (2010), we apply Dirichlet Process Clustering (DPC) in order to carry out inference about the latent classes governing covariance inclusion structures in economic growth regressions. Compared to other methods in the literature (Forgy, 1965; Hartigan and Wong, 1979; Patterson et al., 2002), DPC eliminates the need to set the number of latent classes a priori. While selecting a suitable number of clusters has been a widely discussed problem in the LCA and finite mixture literatures (McLachlan and Peel, 2000, Chap. 6), the nature of Bayesian inference using DPC allows for the automatic selection of an optimal number of clusters for given prior settings.

We assume that $\gamma_{i}$, the $K$-dimensional vector summarizing the variable inclusion profile for model $i$, has elements that arise from a mixture of infinitely, but countably many distributions,

$$
p\left(\gamma_{i}\right)=\sum_{c=1}^{\infty} p\left(g_{i}=c\right) \prod_{k=1}^{K} p\left(\gamma_{i k} \mid g_{i}=c\right),
$$

where $p\left(g_{i}=c\right)$ denotes the probability that model $i$ is assigned to cluster $c$ and $p\left(\gamma_{i k} \mid g_{i}=c\right)$ governs the inclusion probability of the $k$-th covariate in cluster $c$. In turn, for our application we use

$$
\begin{aligned}
p\left(\gamma_{i k} \mid g_{i}=c\right) & \sim \operatorname{Bern}\left(\pi_{c k}\right), \\
\pi_{c k} & \sim \operatorname{Beta}(\delta, \delta), \\
p\left(g_{i}=c\right) & =V_{c} \prod_{j=1}^{c-1}\left(1-V_{j}\right), \\
V_{c} & \sim \operatorname{Beta}(1, \alpha) .
\end{aligned}
$$

Such a mixture model implies, that given assignment to a cluster, the inclusion of covariate $k$ resembles the probabilistic process proposed, for example, in Ley and Steel (2009b). The inclusion probability of covariate $k$ in a given cluster $c$ is thus governed by a Bernoulli distribution whose parameter follows a Beta distribution. The probabilistic structure that governs assignment to the different clusters, $p\left(g_{i}=c\right)$, on the other hand, corresponds to the so-called stick-breaking process formulation of the Dirichlet process (see Sethuraman, 1994; Papaspiliopoulos, 2008; Liverani et al., 
2013). This representation can be interpreted as determining the mixing proportions $p\left(g_{i}=c\right)$ by successive divisions of the unit interval whose relative sizes are determined by independent draws from the $\operatorname{Beta}(1, \alpha)$ distribution.

Posterior inference for DPC can be carried out using MCMC methods. Papaspiliopoulos and Roberts (2008), for instance, present an approach using retrospective sampling. However, identifying a DPC model is difficult due to label switching (Redner and Walker, 1984). We follow Molitor et al. (2010) and derive a suitable partitioning of the set of sampled model profiles using the information on co-assignment to the same clusters during sampling. This information is collapsed into an association matrix that can be interpreted as a similarity matrix between model profiles when assuming that model specifications often assigned to the same cluster are similar. A clustering technique relying only on similarity measures between specification profiles can then be used to find the final clustering, for instance Partitioning Around Medoids (PAM, Kaufman and Rousseeuw, 1990), which is the approach used in our empirical application.

Once a partition has been chosen, several statistics can be used to assess the goodness of fit of the clustering. In our application we rely on measures based on the likelihood ratio chi-squared test statistic $\left(G^{2}\right)$, which measures goodness-of-fit by relating the observed counts of specification profiles in each cluster to the counts predicted by the estimated model. The test statistic is given by $G^{2}=2 \sum_{j}^{2^{K}} q_{j} \ln \frac{q_{j}}{Q_{j}}$, where $q_{j}$ refers to the observed number of counts of specification profile $\gamma_{j}$ and $Q_{j}$ is the expected number of counts assuming independency of the explanatory variables (see for example Brier, 1980). We calculate this $G^{2}$ statistic separately for each cluster and for the aggregated BMA exercise.

In addition, in order to identify substitutability/complementarity of variables based on the cluster solution, we compute a simple measure of interestingness of a variable (IM) in the spirit of the literature on association rules. The interestingness measure $I M$ is determined as the square root of the mean squared deviation of PIPs with respect to the unclustered case across clusters, weighted by the cluster-specific mass of posterior model probabilities. Thus, this measure reflects the stability of the relative importance of the variable across model structures and is able to give an indication of the existence of substitutability/complementarity inclusion patterns across covariates in the model space.

\subsection{A Simulation Exercise}

We assess the performance of the method by making use of a small-scale simulation exercise. We consider a set of ten potential covariates, $x_{k}, k=1, \ldots, 10$ and two settings based on different data generating processes. In the first setting, the dependent variable is a linear combination of the first five covariates and a random error term, $y_{i}=\sum_{k=1}^{5} x_{i k}+\varepsilon_{i}$, where $\varepsilon_{i} \sim N(0,0.01)$ and all covariates are drawn from standard normal distributions. In the second setting, the dependent variable can be represented by two different linear combinations of predictors, so that $y_{i}=\sum_{k=1}^{5} x_{i k}+\varepsilon_{i}=-\sum_{j=6}^{10} x_{i k}+\varepsilon_{i}{ }^{2}$

\footnotetext{
${ }^{2}$ Technically, we implement this setting by defining $x_{i, 10} \approx-\sum_{k=1}^{9} x_{i k}$.
} 
Using simulated datasets with 50 observations for each one of the settings, we perform standard BMA (assuming a single cluster of model specifications) as well as the clustering procedure proposed over the sampled model profiles. We use a Beta-Binomial prior for covariate inclusion (Ley and Steel, 2009b) and a unit information prior for the parameters in the BMA application. For this small example with $K=10$ a complete enumeration of all models is performed. For the clustering procedure, we use a $\operatorname{Gamma}(2,1)$ prior over $\alpha$, elicit $\delta=90$ and retain the top 500 models. The posterior inference is based on 1,500 MCMC iterations, after 1,000 burn-in runs. The results for the first (single cluster) setting are presented in Table 1, where we report the posterior inclusion probabilities and the mean of the posterior distribution of the parameters associated to each one of the covariates, averaged over 100 simulated datasets.

The standard BMA method (see results in panel (a) of Table 1) correctly identifies the covariates included in the true model and the mean of the posterior distribution of the relevant parameters appear very close to the true value of unity. The clustering approach identifies two clusters, with the first one covering over $99 \%$ of the models in the BMA procedure and reproducing the same results as those in the non-clustered case in terms of PIP and means of the posterior distribution of the associated parameters (see panels (b) and (c) in Table 1). In the second setting, whose results are presented in Table 2, the standard BMA procedure averages out the effects of the two alternative data generating processes. The PIP values obtained using BMA are around 0.6 for all variables and the mean of the posterior distribution over the parameters is approximately 0.5 for the first five covariates and -0.5 for the rest of the variables. DPC is able to disentangle the two competing data generating processes, assigning roughly the same posterior mass to each one of the two clusters found. The mean of the posterior distribution of the parameters are in line with the actual values in the true model(s) and the covariates which are not included in the alternative specification have a relatively low PIP and an expected effect which is very close to zero.

\section{Covariate Inclusion Clustering in Economic Growth Regressions}

The clustering method presented in Section 2 is applied to the datasets compiled by Fernandez et al. (2001) and Sala-i Martin et al. (2004) (henceforth, FLS and SDM datasets). These two datasets comprise cross-country information on a large number of potential determinants of income growth and have been extensively used to assess empirically the role played by model uncertainty in economic growth regressions. In addition to GDP per capita growth figures, the FLS dataset is composed by 41 covariates and spans information for 72 countries, while the SDM dataset includes information on 67 different determinants for 88 economies. The variables in both datasets are presented in the Appendix A.

The BMA analysis of both datasets is carried out using a Beta-Binomial prior on covariate inclusion probabilities with a prior average model size of $K / 2$ (20.5 for the FLS dataset and 33.5 for the SDM dataset) and the hyper $g$-prior proposed in Liang et al. (2008) for the regression coefficients. We base our inference concerning the inclusion probability of covariates on five million MCMC model draws, whereby the first two million draws were discarded. Alternatively, we 
Table 1: Simulation Results: Single cluster

(a) Standard BMA

\begin{tabular}{lcr}
\hline & PIP & Post. Mean \\
\hline$x_{1}$ & 1.0000 & 0.9799 \\
$x_{2}$ & 1.0000 & 0.9822 \\
$x_{3}$ & 1.0000 & 0.9810 \\
$x_{4}$ & 1.0000 & 0.9828 \\
$x_{5}$ & 1.0000 & 0.9796 \\
$x_{6}$ & 0.2030 & -0.0001 \\
$x_{7}$ & 0.2053 & -0.0007 \\
$x_{8}$ & 0.2032 & 0.0003 \\
$x_{9}$ & 0.2063 & -0.0002 \\
$x_{10}$ & 0.2053 & -0.0001 \\
\hline
\end{tabular}

(b) DPC: Cluster 1 (>99\%)

\begin{tabular}{lcr}
\hline & PIP & Post. Mean \\
\hline$x_{1}$ & 1.0000 & 0.9799 \\
$x_{2}$ & 1.0000 & 0.9822 \\
$x_{3}$ & 1.0000 & 0.9810 \\
$x_{4}$ & 1.0000 & 0.9828 \\
$x_{5}$ & 1.0000 & 0.9796 \\
$x_{6}$ & 0.2009 & -0.0001 \\
$x_{7}$ & 0.2033 & -0.0007 \\
$x_{8}$ & 0.2011 & 0.0003 \\
$x_{9}$ & 0.2042 & -0.0002 \\
$x_{10}$ & 0.2034 & -0.0001 \\
\hline
\end{tabular}

(c) DPC: Cluster $2(<1 \%)$

\begin{tabular}{ccr}
\hline & PIP & Post. Mean \\
\hline$x_{1}$ & 1.0000 & 0.9799 \\
$x_{2}$ & 1.0000 & 0.9824 \\
$x_{3}$ & 1.0000 & 0.9811 \\
$x_{4}$ & 1.0000 & 0.9825 \\
$x_{5}$ & 1.0000 & 0.9804 \\
$x_{6}$ & 0.9975 & -0.0004 \\
$x_{7}$ & 1.0000 & -0.0025 \\
$x_{8}$ & 1.0000 & 0.0010 \\
$x_{9}$ & 1.0000 & -0.0013 \\
$x_{10}$ & 1.0000 & -0.0010 \\
\hline$y_{i}=\sum_{k=1}^{5} x_{i k}+\varepsilon_{i}$. & Column
\end{tabular}

labelled PIP reports the posterior inclusion probability, column labelled Post. Mean reports the mean of the posterior distribution of the corresponding parameter. See text for details on the setting of the simulation.

also implemented dilution priors over the model space following George (1999) (see also Durlauf et al., 2008). Such a model prior assigns relatively lower prior probability to specifications with highly correlated covariates by weighting the prior model probability using the determinant of the correlation matrix of the explanatory variables. The results obtained using such a dilution prior are not qualitatively different from those with the standard Beta-Binomial prior which are presented below. ${ }^{3}$

Using the top 500 unique models visited by the Markov chain (weighted by their posterior model probability), we apply the clustering procedure described in Section 2 in order to unveil clusters of inclusion patterns across specifications. Technically, we create an auxiliary dataset composed by the 500 top model profiles drawn where the number of observations of each model profile is proportional to its posterior probability. We normalize this auxiliary dataset so that the profile corresponding to the $500^{\text {th }}$ top model is included exactly once and the relative importance of the rest of the models is preserved. For the FLS and SDM dataset the weighted top 500 model profiles in the auxiliary datasets span 33,480 and 28,800 model profile observations, respectively. ${ }^{4}$

Concerning prior elicitation for DPC, we use a setting that implies a relative preference for a smaller number of broad clusters over a multitude of clusters populated by few model structures, which may eventually lack interpretability. We use a $\operatorname{Gamma}(2,1)$ prior over $\alpha$ and $\delta=90$.

\footnotetext{
${ }^{3}$ For the FLS dataset, for instance, the correlation between the posterior inclusion probabilities obtained with the dilution prior and the standard Beta-Binomial prior, as well as between the means and standard deviations of the posterior parameter distributions, tend to be above 0.8. Detailed results of the BMA exercise using George (1999)'s dilution prior are available from the authors upon request.

${ }^{4}$ Expanding the set of top models to cover a larger part of the posterior model probability leads to significant computational complications. For the case of the FLS dataset, which contains less covariates, we also implemented the method for the top 1,000 models, leading to similar results as those presented for the top 500 specifications. Such a result is not very surprising given the fact that the increase in the posterior model probability covered by the top models is very modest when moving from the top 500 to the top 1,000 specifications.
} 
Table 2: Simulation Results: Two clusters

(a) Standard BMA

\begin{tabular}{lcr}
\hline & PIP & Post. Mean \\
\hline$x_{1}$ & 0.6030 & 0.4951 \\
$x_{2}$ & 0.6038 & 0.4949 \\
$x_{3}$ & 0.6032 & 0.4931 \\
$x_{4}$ & 0.6039 & 0.4945 \\
$x_{5}$ & 0.6028 & 0.4941 \\
$x_{6}$ & 0.5983 & -0.4854 \\
$x_{7}$ & 0.5989 & -0.4874 \\
$x_{8}$ & 0.5985 & -0.4853 \\
$x_{9}$ & 0.5983 & -0.4851 \\
$x_{10}$ & 0.5983 & -0.4857 \\
\hline
\end{tabular}

(b) DPC: Cluster $1(49 \%)$

(c) DPC: Cluster $2(51 \%)$

$\overline{\text { Simulation results averaged over } 100}$ simulated datasets. Data generating process: $y_{i} \overline{=\sum_{k=1}^{5} x_{i k}+\varepsilon_{i}=-\sum_{k=6}^{10} x_{i k}+\varepsilon_{i}}$. Column labelled PIP reports the posterior inclusion probability, column labelled Post. Mean reports the mean of the posterior distribution of the corresponding parameter. See text for details on the setting of the simulation.

Posterior inference is based on 1,500 MCMC iterations, after 1,000 burn-in runs. This choice of priors is relatively standard in LCA applications (see e.g. Liverani et al., 2013). ${ }^{5}$

\subsection{Results for the FLS Dataset}

DPC identifies an optimal partition of seven clusters of models by inclusion structure in the FLS dataset. Table 3 provides an overview of the main characteristics of these different model clusters regarding the number of model specifications in the cluster, as well as the mean model size and the average adjusted $R^{2}$ for specifications within the cluster. These statistics are also presented for the unclustered model space considered. Although the top 500 models used for the analysis only cover approximately $8 \%$ of the posterior model probability in the space of potential specifications, the overall unclustered results are very similar to those in Fernandez et al. (2001) concerning the PIP of individual variables. ${ }^{6}$

The first two clusters capture more than half of the posterior mass covered by the set of specifications considered, while clusters 6 and 7 cover a negligible part of the model space in terms of posterior model probability. Cluster 7 is composed by very large models and due to its minimal importance in terms of posterior probability does not appear particularly relevant in terms of interpretation. The cluster-specific $G^{2}$ statistics imply an improvement in fit as compared to the unclustered results once the covariate inclusion structures are assigned to the classes identified.

\footnotetext{
${ }^{5}$ We have carried out several robustness checks changing the elicitation of the priors which did not lead to any significant differences in the inference results as long as the prior setting implies a preference for a relatively small number of clusters.

${ }^{6}$ It should be noted that, in contrast to Fernandez et al. (2001) and Ley and Steel (2007), we employ a hyperprior for prior inclusion probabilities and model-specific parameters, following Ley and Steel (2009b) and Liang et al. (2008), respectively.
} 
The reduction in the $G^{2}$ statistic is very sizable and widespread across the clusters.

Table 3: Summary of FLS clusters

\begin{tabular}{lccllcccc}
\hline & Overall & 1 & 2 & 3 & 4 & 5 & 6 & 7 \\
\hline M Posterior model prob. & 0.08 & 0.03 & 0.02 & 0.01 & 0.01 & 0.01 & 0.00 & 0.00 \\
Average model size & 10.5 & 10.5 & 8.7 & 8.4 & 11.6 & 10.9 & 18.1 & 41.0 \\
Average adjusted $R^{2}$ & 0.83 & 0.84 & 0.81 & 0.80 & 0.85 & 0.84 & 0.90 & 0.91 \\
$G^{2}$ statistic $\left(\times 10^{5}\right)$ & 3.52 & 0.24 & 0.24 & 0.13 & 0.15 & 0.09 & 0.19 & 0.00 \\
\hline
\end{tabular}

Figure 1 offers a graphical representation of the differences in PIPs for individual covariates across the identified clusters. The covariates are sorted by their PIPs in the standard (unclustered) BMA exercise, which are plotted as a solid line together with their corresponding within-cluster PIPs, depicted as bars. It should be noted that the patterns of PIP across variables in all clusters differ structurally from the unclustered BMA results, so that no individual cluster mimics the PIPs obtained by the standard BMA exercise closely. The color of the bars in Figure 1 corresponds to the value of the $I M$ statistic.

Table 4: FLS dataset: Weighted correlation of cluster-specific inclusion for variables with $I M>$ $0.5 \max (I M)$

\begin{tabular}{lccccc}
\hline & SubSahara & EcoOrg & YrsOpen & Muslim & RuleofLaw \\
\hline SubSahara & \multirow{2}{*}{1.00} & 0.50 & -0.65 & -0.34 & 0.73 \\
EcoOrg & & 1.00 & -0.87 & -0.33 & 0.87 \\
YrsOpen & & & 1.00 & 0.45 & -0.96 \\
Muslim & & & & 1.00 & -0.31 \\
RuleofLaw & & & & & 1.00 \\
\hline
\end{tabular}

The PIPs of the four most robust variables of the FLS dataset (Confucian, GDP60, LifeExp and Equipinv) appear stable across clusters. The variables with a higher degree of variability in PIPs across clusters tend to be related to geography (SubSahara), institutions (EcoOrg, RuleofLaw and YrsOpen ${ }^{7}$ ) and religion (Muslim). The characteristics of the inclusion structure of these variables across clusters can be best understood by computing the weighted correlation matrix of cluster-specific binary inclusion variables, which is presented in Table 4. The correlation among covariate inclusion variables reveals that SubSahara, EcoOrg and RuleofLaw tend to contain complementary information in the sense of appearing together in specifications. The same is true for the group of variables formed by YrsOpen and Muslim, while the inclusion of these two sets of variables presents sizable negative correlation. This result indicates that some of the effects of

${ }^{7}$ The variable YrsOpen is based on the Sachs-Warner index of openness, which has a strong institutional component. For example, socialist economies are automatically considered closed to trade by this indicator. 


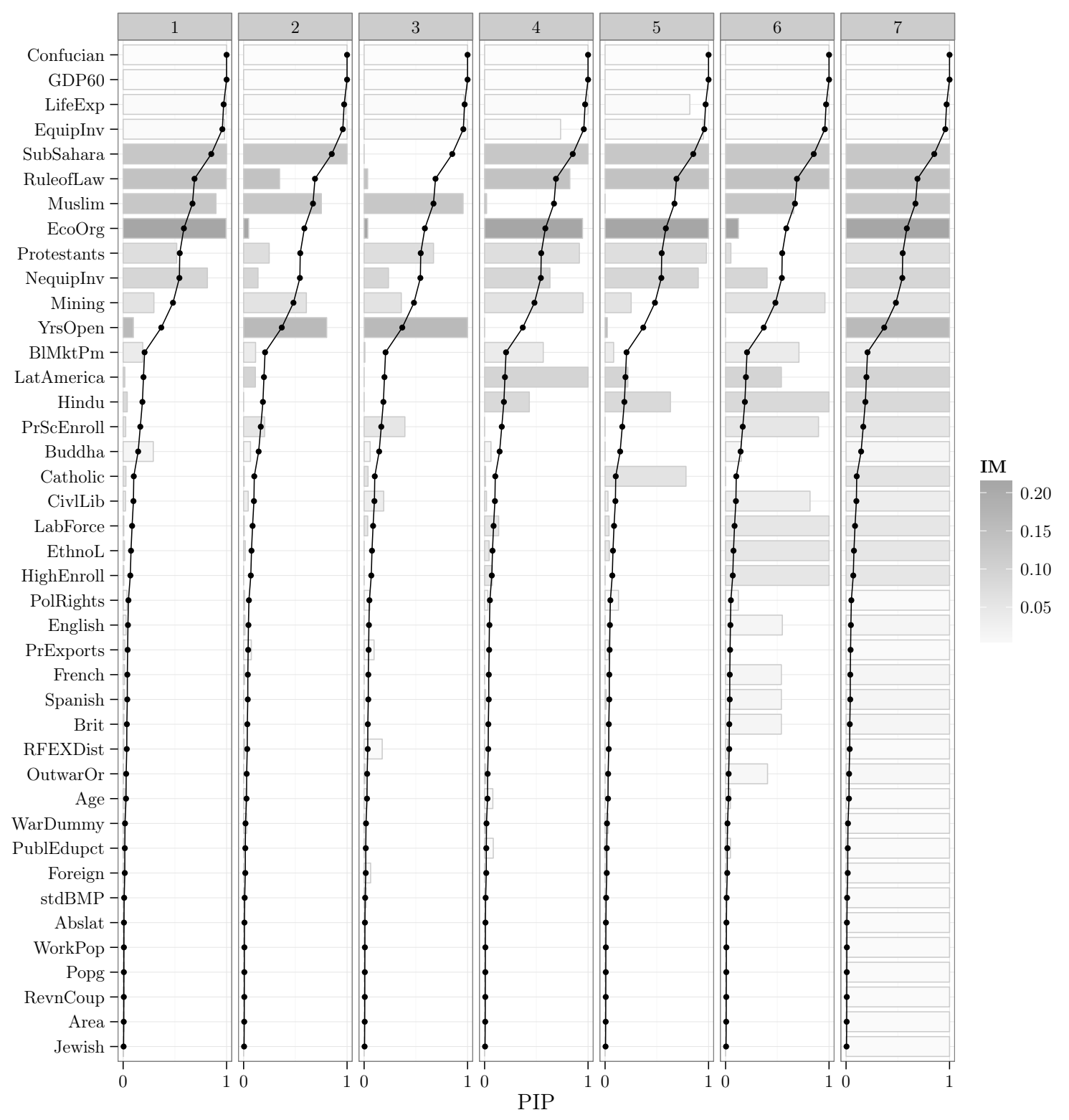

Figure 1: FLS dataset: PIPs in unclustered BMA (solid line) and by identified cluster (bars) 
institutions and geographical variables on economic growth can be alternatively modeled using these two groups of covariates in a robust manner, but that once that they are controlled for, the inclusion of variables of the other group appears redundant.

The interplay of changes in PIPs across clusters presented in Figure 1 indicates that the set of religious, institutional and geographical variables used in cross-country growth regressions often contain redundant information which can be replicated using different subgroups of them. An example of such a phenomenon is observed when comparing clusters 1 and 3 . The importance of SubSahara and RuleofLaw as growth determinants which can be inferred from the results in cluster 1 disappears in cluster 3 and their fall in PIPs occurs in parallel to a strong increase in PIP for YrsOpen. The set of religious variables (Muslim, Catholic, Protestants, Hindu and, to a lesser extent, Buddha) also presents large variation in PIPs across clusters.

\subsection{Results for the SDM Dataset}

Ley and Steel (2009a) found very weak (bivariate and/or trivariate) jointness in the group of covariates included in the SDM dataset. Our procedure splits the model space into three different model clusters by covariate inclusion patterns. Table 5 presents the summary statistics for the identified clusters. The top 500 unique specifications cover $40 \%$ of the posterior model probability, a much larger proportion than in the case of the FLS dataset. The structure of variable inclusion for the SDM dataset appears to have a different nature as compared to the results for the FLS dataset. In addition to the lower number of identified clusters, the first two classes of inclusion structures identified exhibit relatively similar characteristics in terms of the posterior model probability covered. As in the case of the FLS dataset, the cluster specific $G^{2}$ statistics are lower than the corresponding value for the model without clustering, thus supporting the method employed.

Figure 2 depicts the PIPs of the variables in the SDM dataset computed using the top 500 models, as well as those derived from the models in the single clusters. ${ }^{8}$ The results show a large degree of variability in PIPs across clusters for many of the covariates, including those presenting the highest PIPs in the unclustered case.

Table 5: Summary of SDM clusters

\begin{tabular}{lclll}
\hline & Overall & 1 & 2 & 3 \\
\hline M Posterior model prob. & 0.40 & 0.21 & 0.17 & 0.03 \\
Average model size & 5.5 & 6.5 & 3.9 & 6.8 \\
Average adjusted $R^{2}$ & 0.67 & 0.71 & 0.62 & 0.70 \\
$G^{2}$ statistic $\left(\times 10^{5}\right)$ & 10.25 & 1.33 & 2.36 & 0.58 \\
\hline
\end{tabular}

\footnotetext{
${ }^{8}$ Variables with PIP lower than $5 \%$ have been excluded in order to improve the readability of the graph. For these variables no remarkable changes could be detected when comparing the BMA results with the cluster-specific PIPs.
} 


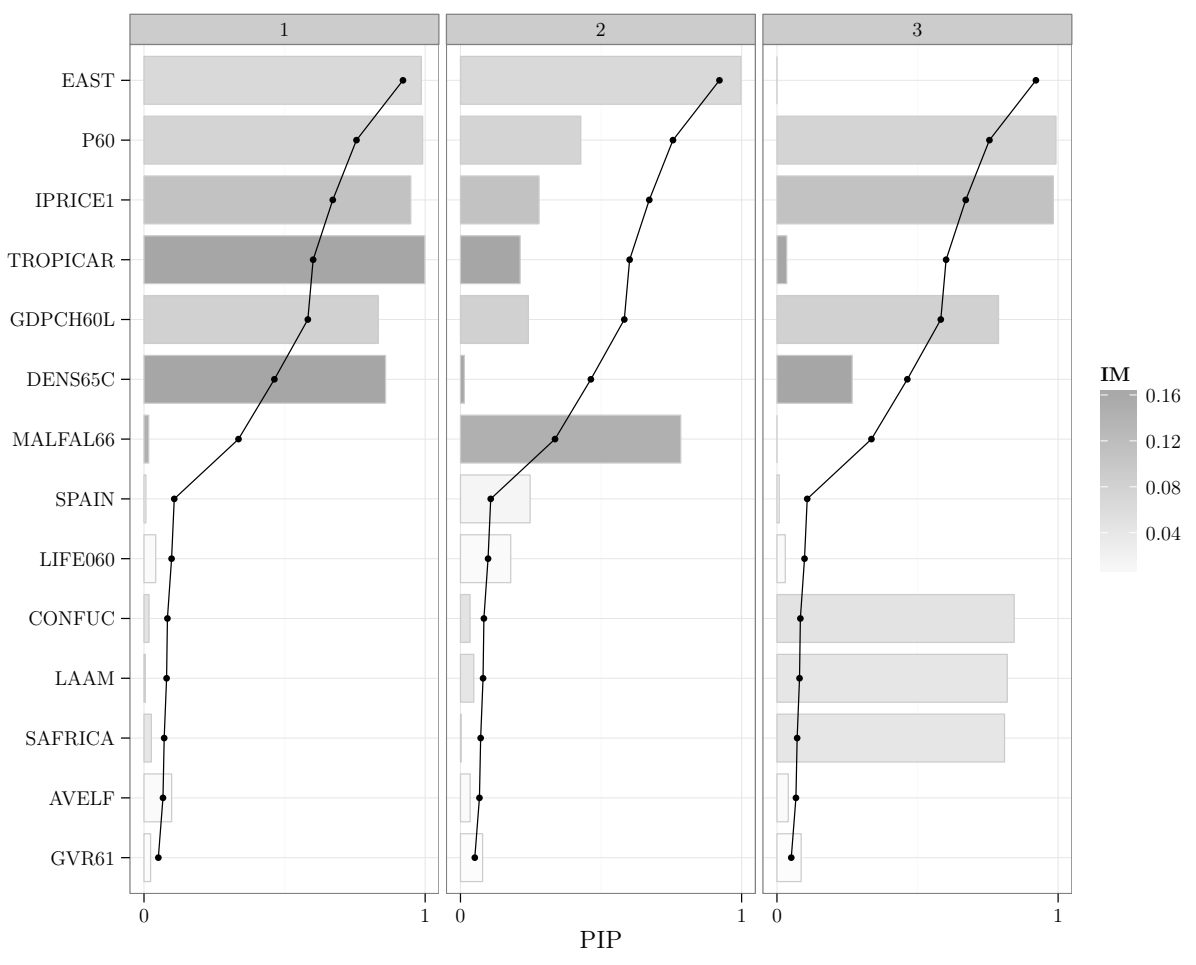

Figure 2: SDM dataset: PIPs in unclustered BMA (solid line) and by identified cluster (bars)

Given the large posterior probability mass over models covered by the first two clusters, we concentrate on the differences in PIPs observed between these two. Remarkable differences in PIPs across these two clusters can be observed for the MALFAL66 variable, which presents a much higher PIP in the second cluster, making it the second most important variable for models within that cluster. Such a phenomenon is accompanied by a sizable decrease in PIP for P60, IPRICE1, TROPICAR, GDPCH60L and DENS65C. The empirical literature on model uncertainty in cross-country growth regressions which analyzes the SDM dataset often reports on the effect that the use of different approaches to parameter shrinkage has on the importance of MALFAL66. Schneider and Wagner (2012) as well as Hofmarcher et al. (2014), for instance, find that the robustness of MALFAL66 as a determinant of income growth differences across countries improves when estimation methods based on LASSO and elastic nets are used. In addition, the results in Schneider and Wagner (2012) and Hofmarcher et al. (2014) also indicate a loss of importance of DENS65C when methods implying a more stringent shrinkage are used in the estimation. These are precisely two of the variables which present the highest values of $I M$ in our results, hinting to the fact that their relative importance depends on the type of model (as represented by the variable inclusion structure cluster) considered.

Such a pattern of substitutability across covariates is easily recognizable from the weighted correlation matrix of cluster-specific binary inclusion variables for the group of variables with the highest $I M$ values, which is presented in Table 6 . The correlation patterns present in the model space indicate that MALFAL66 tends to act as a substitute of the group of variables composed by IPRICE1, TROPICAR, GDPCH60L and DENS65C. The difference in average model size across these 
Table 6: SDM dataset: Weighted correlation of cluster-specific inclusion for variables with $I M>$ $0.5 \max (I M)$

\begin{tabular}{lccccc}
\hline & DENS65C & GDPCH60L & IPRICE1 & MALFAL66 & TROPICAR \\
\hline DENS65C & 1.00 & 0.95 & 0.92 & -0.93 & 0.97 \\
GDPCH60L & & 1.00 & 1.00 & -1.00 & 0.83 \\
IPRICE1 & & & 1.00 & -1.00 & 0.79 \\
MALFAL66 & & & & 1.00 & -0.80 \\
TROPICAR & & & & & 1.00 \\
\hline
\end{tabular}

two important clusters in the space of posterior inclusion probability structures is in line with the strong impact of different parameter shrinkage approaches on the relative importance of the variables which is highlighted in previous literature. In addition, in their study of pairwise jointness measures, Doppelhofer and Weeks (2009a) report that P60, IPRICE1, DENS65C and TROPICAR exhibit significant negative bivariate jointness with MALFAL66, a result that can be easily reconciled with the output of our analysis. While Ley and Steel (2007) find very limited evidence for jointness structures in the SDM dataset, the only triplets of important variables for which disjointness is reported also involve TROPICAR and MALFAL66.

In spite of the fact that the third cluster that DPC identifies covers a very small part of the posterior mass over models, its PIP structure also reveals interesting patterns as compared to the other two clusters. In this group of models, two of the most relevant variables in terms of (unclustered) PIP, EAST and TROPICAR, lose their importance and their information is captured by a different set of geographical and religious variables (CONFUC, LAAM and SAFRICA). The results in Doppelhofer and Weeks (2009a) concerning the complementarity of EAST and TROPICAR and the substitutability of EAST with respect to CONFUC, LAAM and SAFRICA are perfectly in line with these results. In addition, Doppelhofer and Weeks (2009a) find the latter to be complements, which is also supported by the comparison of the PIPs in our third cluster with those in the other two.

\section{Conclusions and Future Paths of Research}

In this contribution we are concerned with covariate inclusion patterns of BMA exercises with large model spaces. Recent research on such jointness structures tends to choose a low-dimensional approach to such an analysis and thus concentrates on bivariate or trivariate approaches, by calculating the inclusion relationships of few explaining factors at a time. We propose a novel approach by utilizing LCA techniques and apply DPC to two well known datasets in the BMA growth literature. The clustering method put forward in our contribution aims at unveiling commonalities in the joint inclusion of variables and thus offering the applied econometrician evidence about the competing structures (as formed by groups of variables that appear together) that are covered by the posterior over the model space. 
Our results indicate that within the set of models sampled by the Markov chain in the BMA analysis of determinants of economic growth, several distinct clusters of models by covariate inclusion can be identified. For the FLS data, we identify seven clusters of models which differ in the inclusion structure for geographic, institutional and religious covariates. In contrast, the SDM dataset only reveals three latent classes with very different dependency structures. The inclusion of the variable measuring malaria prevalence is shown to vary strongly across clusters, with its effect on economic growth being captured often by other factors such as the fraction of tropical area and coastal population density.

We show that the study of dependency structures in covariate inclusion for large model spaces appears particularly relevant in order to understand the nature of the factors affecting global patterns of income growth. The proposed method lends itself to further straightforward expansions such as the use of low-dimensional jointness measures for the analysis of within-cluster inclusion patterns for small groups of covariates. The assessment of covariate inclusion clusters in the model space under different shrinkage priors can also shed light on the effects of multicollinearity on the robustness of economic growth determinants to model uncertainty.

In order to make our method and results comparable to those in the literature on jointness measures, we decided to follow a two-step procedure and use the clustering method on the model profiles visited by the Markov chain of the BMA procedure. The LCA and DPC methods proposed in this contribution would also lend themselves to create priors over suitable covariate combinations in the specifications that compose the model space. This path of further research, which we are pursuing at the moment, appears particularly promising in order to unify the literature on jointness and dilution priors in BMA applications. 


\section{References}

Blaydes, L. and Linzer, D. A. (2008). The political economy of women's support for fundamentalist Islam. World Politics, 60(04):576-609.

Breen, R. (2000). Why is support for extreme parties underestimated by surveys? A latent class analysis. British Journal of Political Science, 30(02):375-382.

Brier, S. S. (1980). Analysis of contingency tables under cluster sampling. Biometrika, 67(3):591-596.

Brock, W. A. and Durlauf, S. N. (2001). What have we learned from a decade of empirical research on growth? Growth empirics and reality. The World Bank Economic Review, 15(2):229-272.

Doppelhofer, G. and Weeks, M. (2009a). Jointness of growth determinants. Journal of Applied Econometrics, 24(2):209-244.

Doppelhofer, G. and Weeks, M. (2009b). Jointness of growth determinants: Reply to comments by Rodney Strachan, Eduardo Ley and Mark FJ Steel. Journal of Applied Econometrics, $24(2): 252-256$.

Doppelhofer, G., Weeks, M., et al. (2005). Jointness of determinants of economic growth. In Money Macro and Finance (MMF) Research Group Conference 2005, number 54. Money Macro and Finance Research Group.

Durlauf, S. N., Kourtellos, A., and Tan, C. M. (2008). Are any growth theories robust? The Economic Journal, 118(527):329-346.

Fernandez, C., Ley, E., and Steel, M. F. J. (2001). Model uncertainty in cross-country growth regressions. Journal of Applied Econometrics, 16(5):563-576.

Forgy, E. W. (1965). Cluster analysis of multivariate data: efficiency versus interpretability of classifications. Biometrics, 21:768-769.

George, E. (1999). Discussion of Bayesian model averaging and model search strategies by M.A. Clyde. In Bernardo, J., Berger, J., Dawid, A., and Smith, A., editors, Bayesian Statistics 6, pages $157-177$.

Glass, D. H. (2013). Confirmation measures of association rule interestingness. Knowledge-Based Systems, 44:65-77.

Hartigan, J. A. and Wong, M. A. (1979). Algorithm AS 136: A k-means clustering algorithm. Applied Statistics, 28(1):100-108.

Hoeting, J. A., Madigan, D., Raftery, A. E., and Volinsky, C. T. (1999). Bayesian model averaging: A tutorial. Statistical Science, 14(4):382-401. 
Hofmarcher, P., Crespo Cuaresma, J., Grün, B., and Hornik, K. (2014). Last night a shrinkage saved my life: Economic growth, model uncertainty and correlated regressors. Mimeo, Vienna University of Economics and Business.

Kaufman, L. and Rousseeuw, P. J. (1990). Finding Groups in Data. John Wiley \& Sons, Inc., New York, USA.

Lazarsfeld, P. F. (1950). The logical and mathematical foundations of latent structure analysis. In Stouffer, S. A., editor, Measurement and Prediction, pages 362-412. John Wiley \& Sons, New York.

Ley, E. and Steel, M. F. (2007). Jointness in Bayesian variable selection with applications to growth regression. Journal of Macroeconomics, 29(3):476-493.

Ley, E. and Steel, M. F. (2009a). Comments on 'Jointness of growth determinants'. Journal of Applied Econometrics, 24(2):248-251.

Ley, E. and Steel, M. F. (2009b). On the effect of prior assumptions in Bayesian model averaging with applications to growth regression. Journal of Applied Econometrics, 24(4):651-674.

Liang, F., Paulo, R., Molina, G., Clyde, M. A., and Berger, J. O. (2008). Mixtures of g priors for Bayesian variable selection. Journal of the American Statistical Association, 103(481).

Liverani, S., Hastie, D. I., Papathomas, M., and Richardson, S. (2013). PReMiuM: An R package for profile regression mixture models using Dirichlet processes. arXiv preprint arXiv:1303.2836.

Madigan, D. and York, J. (1995). Bayesian graphical models for discrete data. International Statistical Review, 63:215-232.

Masanjala, W. and Papageorgiou, C. (2008). Rough and lonely road to prosperity: A reexamination of the sources of growth in Africa using Bayesian model averaging. Journal of Applied Econometrics, 23:671-682.

McLachlan, G. J. and Peel, D. (2000). Finite Mixture Models. Wiley.

Molitor, J., Papathomas, M., Jerrett, M., and Richardson, S. (2010). Bayesian profile regression with an application to the national survey of children's health. Biostatistics, 11(3):484-498.

Papaspiliopoulos, O. (2008). A note on posterior sampling from Dirichlet mixture models. Technical Report 8, CRISM Paper.

Papaspiliopoulos, O. and Roberts, G. (2008). Retrospective Markov chain Monte Carlo methods for Dirichlet process hierarchical models. Biometrika, 95:169-186.

Patterson, B. H., Dayton, C. M., and Graubard, B. I. (2002). Latent class analysis of complex sample survey data: Application to dietary data. Journal of the American Statistical Association, 97(459):721-741. 
Redner, R. A. and Walker, H. F. (1984). Mixture densities, maximum likelihood and the EM algorithm. SIAM Review, 26(2):195-239.

Sala-i Martin, X., Doppelhofer, G., and Miller, R. I. (2004). Determinants of long-term growth: A Bayesian averaging of classical estimates (BACE) approach. American Economic Review, 94(4):813-835.

Schneider, U. and Wagner, M. (2012). Catching growth determinants with the adaptive LASSO. German Economic Review, 13:71-85.

Sethuraman, J. (1994). A constructive definition of Dirichlet priors. Statistica Sinica, 4:639-650.

Strachan, R. W. (2009). Comment on 'Jointness of growth determinants' by Gernot Doppelhofer and Melvyn Weeks. Journal of Applied Econometrics, 24(2):245-247.

Tan, P.-N., Kumar, V., and Srivastava, J. (2004). Selecting the right objective measure for association analysis. Information Systems, 29(4):293-313.

Vermunt, J. K. and Magidson, J. (2002). Latent class cluster analysis. In Hagenaars, J. and McCutcheon, A., editors, Applied Latent Class Analysis, pages 89-106. Cambridge University Press, Cambridge, UK. 


\section{A. Datasets}

Table A.1: Variable names and descriptive statistics - FLS

\begin{tabular}{|c|c|c|c|c|}
\hline & Abbreviation & Variable & Mean & Std. Dev \\
\hline 1 & Abslat & Absolute latitude & 25.73 & 17.250 \\
\hline 2 & Age & Age & 23.71 & 37.307 \\
\hline 3 & Area & Area (Scale Effect) & 972.92 & 2051.976 \\
\hline 4 & BlMktPm & Black Market Premium & 0.16 & 0.291 \\
\hline 5 & Brit & British Colony dummy & 0.32 & 0.470 \\
\hline 6 & Buddha & Fraction Buddhist & 0.06 & 0.184 \\
\hline 7 & Catholic & Fraction Catholic & 0.42 & 0.397 \\
\hline 8 & CivlLib & Civil Liberties & 3.47 & 1.712 \\
\hline 9 & Confucian & Fraction Confucian & 0.02 & 0.087 \\
\hline 10 & EcoOrg & Degree of Capitalism & 3.54 & 1.266 \\
\hline 11 & English & Fraction of Pop. Speaking English & 0.08 & 0.239 \\
\hline 12 & EquipInv & Equipment investment & 0.04 & 0.035 \\
\hline 13 & EthnoL & Ethnolinguistic fractionalization & 0.37 & 0.296 \\
\hline 14 & Foreign & Fraction speaking foreign language & 0.37 & 0.422 \\
\hline 15 & French & French Colony dummy & 0.12 & 0.333 \\
\hline 16 & GDP60 & GDP level in 1960 & 7.49 & 0.885 \\
\hline 17 & HighEnroll & Higher education enrollment & 0.04 & 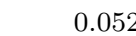 \\
\hline 18 & Hindu & Fraction Hindu & 0.02 & 0.101 \\
\hline 19 & Jewish & Fraction Jewish & 0.01 & 0.097 \\
\hline 20 & LabForce & Size labor force & 9305.38 & 24906.056 \\
\hline 21 & LatAmerica & Latin American dummy & 0.28 & 0.451 \\
\hline 22 & LifeExp & Life expectancy & 56.58 & 11.448 \\
\hline 23 & Mining & Fraction GDP in mining & 0.04 & 0.077 \\
\hline 24 & Muslim & Fraction Muslim & 0.15 & 0.295 \\
\hline 25 & NequipInv & Non-Equipment Investment & 0.15 & 0.055 \\
\hline 26 & OutwarOr & Outward Orientation & 0.39 & 0.491 \\
\hline 27 & PolRights & Political Rights & 3.45 & 1.896 \\
\hline 28 & Popg & Population Growth & 0.02 & 0.010 \\
\hline 29 & PrExports & Primary exports, 1970 & 0.67 & 0.299 \\
\hline 30 & Protestants & Fraction Protestant & 0.17 & 0.252 \\
\hline 31 & PrScEnroll & Primary School Enrollment, 1960 & 0.80 & 0.246 \\
\hline 32 & PublEdupct & Public Education Share & 0.02 & 0.009 \\
\hline 33 & RevnCoup & Revolutions and coups & 0.18 & 0.238 \\
\hline 34 & RFEXDist & Exchange rate distortions & 121.71 & 41.001 \\
\hline 35 & RuleofLaw & Rule of law & 0.55 & 0.335 \\
\hline 36 & Spanish & Spanish Colony dummy & 0.22 & 0.419 \\
\hline 37 & stdBMP & SD of black-market premium & 45.60 & 95.802 \\
\hline 38 & SubSahara & Sub-Saharan dummy & 0.21 & 0.409 \\
\hline 39 & WarDummy & War dummy & 0.40 & 0.494 \\
\hline 40 & WorkPop & Ratio workers to population & -0.95 & 0.189 \\
\hline 41 & $\mathrm{y}$ & GDP per capita growth & 0.02 & 0.018 \\
\hline 42 & YrsOpen & Number of Years open economy & 0.44 & 0.355 \\
\hline
\end{tabular}


Table A.2: Variable names and descriptive statistics - SDM

\begin{tabular}{|c|c|c|c|c|}
\hline & Abbreviation & Variable & Mean & Std. Dev. \\
\hline 1 & ABSLATIT & Absolute latitude & 23.21 & 16.843 \\
\hline 2 & AIRDIST & Air distance to big cities & 4324.17 & 2613.763 \\
\hline 3 & AVELF & Ethnolinguistic fractionalization & 0.35 & 0.302 \\
\hline 4 & BRIT & British colony & 0.32 & 0.468 \\
\hline 5 & BUDDHA & Fraction Buddhist & 0.05 & 0.168 \\
\hline 6 & CATHOO & Fraction Catholic & 0.33 & 0.415 \\
\hline 7 & CIV72 & Civil liberties & 0.51 & 0.326 \\
\hline 8 & COLONY & Colony dummy & 0.75 & 0.435 \\
\hline 9 & CONFUC & Fraction Confucian & 0.02 & 0.079 \\
\hline 10 & DENS60 & Population density costal 1960's & 108.07 & 201.445 \\
\hline 11 & DENS65C & Population density 1960 & 146.87 & 509.828 \\
\hline 12 & DENS65I & Interior density & 43.37 & 88.063 \\
\hline 13 & DPOP6090 & Population growth rate $1960-1990$ & 0.02 & 0.009 \\
\hline 14 & EAST & East Asian dummy & 0.11 & 0.319 \\
\hline 15 & ECORG & Capitalism & 3.47 & 1.381 \\
\hline 16 & ENGFRAC & English-speaking population & 0.08 & 0.252 \\
\hline 17 & EUROPE & European dummy & 0.22 & 0.414 \\
\hline 18 & FERTLDC1 & Fertility in 1960's & 1.56 & 0.419 \\
\hline 19 & GDE1 & Defense spending share & 0.03 & 0.025 \\
\hline 20 & GDPCH60L & GDP $1960(\log )$ & 7.35 & 0.901 \\
\hline 21 & GEEREC1 & Public education spending share in GDP in 1960's & 0.02 & 0.010 \\
\hline 22 & GGCFD3 & Government consumption share deflated with GDP prices & 0.05 & 0.039 \\
\hline 23 & GOVNOM1 & Nominal government GDP share 1960's & 0.15 & 0.058 \\
\hline 24 & GOVSH61 & Government share of GDP & 0.17 & 0.071 \\
\hline 25 & GR6096 & Average growth rate of GDP per capita 1960-1996 & 0.02 & 0.019 \\
\hline 26 & GVR61 & Government consumption share 1960's & 0.12 & 0.075 \\
\hline 27 & H60 & Higher education in 1960 & 0.04 & 0.050 \\
\hline 28 & HERF00 & Religous intensity & 0.78 & 0.193 \\
\hline 29 & HINDU00 & Fraction Hindu & 0.03 & 0.125 \\
\hline 30 & IPRICE1 & Investment price & 92.47 & 53.678 \\
\hline 31 & LAAM & Latin American dummy & 0.23 & 0.421 \\
\hline 32 & LANDAREA & Land area & 867188.52 & 1814688.290 \\
\hline 33 & LANDLOCK & Landlocked country dummy & 0.17 & 0.378 \\
\hline 34 & LHCPC & Hydrocarbon deposits in 1993 & 0.42 & 4.351 \\
\hline 35 & LIFE060 & Life expectancy in 1960 & 53.72 & 12.062 \\
\hline 36 & LT100CR & Fraction of land area near navigable water & 0.47 & 0.380 \\
\hline 37 & MALFAL66 & Malaria prevalence in 1960 's & 0.34 & 0.431 \\
\hline 38 & MINING & Fraction GDP in mining & 0.05 & 0.077 \\
\hline 39 & MUSLIM00 & Fraction Muslim & 0.15 & 0.296 \\
\hline 40 & NEWSTATE & Time of independence & 1.01 & 0.977 \\
\hline 41 & OIL & Oil-producing country dummy & 0.06 & 0.233 \\
\hline 42 & OPENDEC1 & (Imports+exports)/GDP & 0.52 & 0.336 \\
\hline 43 & ORTHOO & Fraction Orthodox & 0.02 & 0.098 \\
\hline 44 & OTHFRAC & Fraction speaking foreign language & 0.32 & 0.414 \\
\hline 45 & $\mathrm{P} 60$ & Primary schooling 1960 & 0.73 & 0.293 \\
\hline 46 & PI6090 & Average inflation 1960-1990 & 13.13 & 14.990 \\
\hline 47 & POP1560 & Fraction population less than 15 & 0.39 & 0.075 \\
\hline 48 & POP60 & Population in 1960 & 20308.08 & 52538.387 \\
\hline 49 & POP6560 & Fraction population over 65 & 0.05 & 0.029 \\
\hline 50 & PRIEXP70 & Primary exports in 1970 & 0.72 & 0.283 \\
\hline 51 & PRIGHTS & Political rights & 3.82 & 1.997 \\
\hline 52 & PROT00 & Fraction Protestant & 0.14 & 0.285 \\
\hline 53 & RERD & Real exchange rate distortions & 125.03 & 41.706 \\
\hline 54 & REVCOUP & Revolution and coups & 0.18 & 0.232 \\
\hline 55 & SAFRICA & African dummy & 0.31 & 0.464 \\
\hline 56 & SCOUT & Outward orientation & 0.40 & 0.492 \\
\hline 57 & SIZE60 & Size of the economy & 16.15 & 1.820 \\
\hline 58 & SOCIALIST & Socialist dummy & 0.07 & 0.254 \\
\hline 59 & SPAIN & Spanish colony & 0.17 & 0.378 \\
\hline 60 & SQPI6090 & Square of inflation 1960-1990 & 394.54 & 1119.699 \\
\hline 61 & TOT1DEC1 & Terms of trade growth in 1960's & 0.00 & 0.035 \\
\hline 62 & TOTIND & Terms of trade ranking & 0.28 & 0.190 \\
\hline 63 & TROPICAR & Fraction of tropical area & 0.57 & 0.472 \\
\hline 64 & TROPPOP & Fraction population in tropics & 0.30 & 0.373 \\
\hline 65 & WARTIME & Fraction spent in war $1960-1990$ & 0.07 & 0.152 \\
\hline 66 & WARTORN & War participation 1960-1990 & 0.40 & 0.492 \\
\hline 67 & YRSOPEN & Years open & 0.36 & 0.344 \\
\hline 68 & ZTROPICS & Tropical climate zone & 0.19 & 0.269 \\
\hline
\end{tabular}




\section{B. Posterior Inclusion Probabilities by Cluster}

Table B.1: PIPs within detected clusters - FLS

\begin{tabular}{|c|c|c|c|c|c|c|c|c|c|}
\hline & Overall & 1 & 2 & 3 & 4 & 5 & 6 & 7 & $\mathrm{IM}$ \\
\hline GDP level in 1960 & 1.00 & 1.00 & 1.00 & 1.00 & 1.00 & 1.00 & 1.00 & 1.00 & 0.00 \\
\hline Fraction Confucian & 1.00 & 1.00 & 1.00 & 1.00 & 1.00 & 1.00 & 1.00 & 1.00 & 0.00 \\
\hline Life expectancy & 0.97 & 0.99 & 0.99 & 0.97 & 1.00 & 0.82 & 1.00 & 1.00 & 0.00 \\
\hline Equipment investment & 0.96 & 0.98 & 1.00 & 1.00 & 0.73 & 0.95 & 1.00 & 1.00 & 0.01 \\
\hline Sub-Saharan dummy & 0.85 & 1.00 & 1.00 & 0.00 & 1.00 & 1.00 & 1.00 & 1.00 & 0.13 \\
\hline Rule of law & 0.69 & 1.00 & 0.35 & 0.03 & 0.82 & 1.00 & 1.00 & 1.00 & 0.14 \\
\hline Fraction Muslim & 0.67 & 0.90 & 0.75 & 0.96 & 0.02 & 0.00 & 0.66 & 1.00 & 0.13 \\
\hline Degree of Capitalism & 0.59 & 0.99 & 0.05 & 0.04 & 0.95 & 1.00 & 0.12 & 1.00 & 0.21 \\
\hline Fraction Protestant & 0.55 & 0.52 & 0.25 & 0.67 & 0.92 & 0.98 & 0.05 & 1.00 & 0.07 \\
\hline Non-Equipment Investment & 0.54 & 0.81 & 0.14 & 0.24 & 0.63 & 0.90 & 0.40 & 1.00 & 0.09 \\
\hline Fraction GDP in mining & 0.48 & 0.30 & 0.61 & 0.36 & 0.95 & 0.25 & 0.96 & 1.00 & 0.06 \\
\hline Number of Years open economy & 0.37 & 0.10 & 0.80 & 1.00 & 0.00 & 0.02 & 0.00 & 1.00 & 0.16 \\
\hline Black Market Premium & 0.21 & 0.19 & 0.12 & 0.01 & 0.57 & 0.08 & 0.71 & 1.00 & 0.04 \\
\hline Latin American dummy & 0.20 & 0.02 & 0.11 & 0.00 & 1.00 & 0.22 & 0.54 & 1.00 & 0.10 \\
\hline Fraction Hindu & 0.19 & 0.04 & 0.00 & 0.00 & 0.43 & 0.63 & 1.00 & 1.00 & 0.09 \\
\hline Primary School Enrollment, 1960 & 0.17 & 0.02 & 0.20 & 0.39 & 0.00 & 0.00 & 0.90 & 1.00 & 0.05 \\
\hline Fraction Buddhist & 0.15 & 0.29 & 0.07 & 0.06 & 0.06 & 0.00 & 0.15 & 1.00 & 0.02 \\
\hline Fraction Catholic & 0.10 & 0.03 & 0.01 & 0.04 & 0.01 & 0.78 & 0.00 & 1.00 & 0.06 \\
\hline Civil Liberties & 0.10 & 0.02 & 0.04 & 0.19 & 0.02 & 0.03 & 0.82 & 1.00 & 0.04 \\
\hline Size labor force & 0.09 & 0.01 & 0.01 & 0.04 & 0.14 & 0.04 & 1.00 & 1.00 & 0.05 \\
\hline Ethnolinguistic fractionalization & 0.08 & 0.01 & 0.02 & 0.00 & 0.04 & 0.04 & 1.00 & 1.00 & 0.05 \\
\hline Higher education enrollment & 0.07 & 0.01 & 0.00 & 0.00 & 0.08 & 0.00 & 1.00 & 1.00 & 0.05 \\
\hline Political Rights & 0.05 & 0.04 & 0.01 & 0.06 & 0.03 & 0.13 & 0.12 & 1.00 & 0.00 \\
\hline Fraction of Pop. Speaking English & 0.05 & 0.03 & 0.01 & 0.00 & 0.00 & 0.00 & 0.55 & 1.00 & 0.02 \\
\hline Primary exports, 1970 & 0.04 & 0.02 & 0.07 & 0.10 & 0.00 & 0.03 & 0.00 & 1.00 & 0.00 \\
\hline French Colony dummy & 0.04 & 0.01 & 0.01 & 0.00 & 0.00 & 0.00 & 0.54 & 1.00 & 0.02 \\
\hline Spanish Colony dummy & 0.04 & 0.01 & 0.00 & 0.00 & 0.01 & 0.01 & 0.54 & 1.00 & 0.02 \\
\hline British Colony dummy & 0.04 & 0.01 & 0.00 & 0.00 & 0.00 & 0.00 & 0.54 & 1.00 & 0.02 \\
\hline Exchange rate distortions & 0.03 & 0.01 & 0.01 & 0.17 & 0.00 & 0.00 & 0.02 & 1.00 & 0.01 \\
\hline Outward Orientation & 0.03 & 0.00 & 0.00 & 0.01 & 0.01 & 0.00 & 0.41 & 1.00 & 0.01 \\
\hline Age & 0.03 & 0.02 & 0.02 & 0.02 & 0.08 & 0.00 & 0.05 & 1.00 & 0.00 \\
\hline War dummy & 0.02 & 0.01 & 0.03 & 0.00 & 0.01 & 0.03 & 0.02 & 1.00 & 0.00 \\
\hline Public Education Shar & 0.02 & 0.00 & 0.00 & 0.00 & 0.08 & 0.00 & 0.05 & 1.00 & 0.00 \\
\hline Fraction speaking foreign language & 0.02 & 0.01 & 0.00 & 0.06 & 0.00 & 0.00 & 0.00 & 1.00 & 0.00 \\
\hline SD of black-market premium & 0.01 & 0.00 & 0.01 & 0.02 & 0.00 & 0.00 & 0.00 & 1.00 & 0.00 \\
\hline Absolute latitude & 0.01 & 0.01 & 0.00 & 0.01 & 0.00 & 0.00 & 0.00 & 1.00 & 0.00 \\
\hline Ratio workers to population & 0.01 & 0.01 & 0.00 & 0.01 & 0.00 & 0.00 & 0.00 & 1.00 & 0.00 \\
\hline Population Growth & 0.01 & 0.01 & 0.00 & 0.00 & 0.00 & 0.00 & 0.00 & 1.00 & 0.00 \\
\hline Revolutions and coups & 0.01 & 0.01 & 0.00 & 0.00 & 0.00 & 0.00 & 0.00 & 1.00 & 0.00 \\
\hline cale Effect) & 0.01 & 0.01 & 0.00 & 0.00 & 0.00 & 0.00 & 0.00 & 1.00 & 0.00 \\
\hline Fraction Jewish & 0.00 & 0.00 & 0.00 & 0.00 & 0.00 & 0.00 & 0.00 & 1.00 & 0.00 \\
\hline
\end{tabular}


Table B.2: PIPs within detected clusters - SDM

\begin{tabular}{|c|c|c|c|c|c|}
\hline & Overall & 1 & 2 & 3 & IM \\
\hline East Asian dummy & 0.92 & 0.99 & 1.00 & 0.00 & 0.06 \\
\hline Primary schooling 1960 & 0.76 & 0.99 & 0.43 & 0.99 & 0.08 \\
\hline Investment price & 0.67 & 0.95 & 0.28 & 0.98 & 0.11 \\
\hline Fraction of tropical area & 0.60 & 1.00 & 0.21 & 0.03 & 0.17 \\
\hline GDP $1960(\log )$ & 0.58 & 0.83 & 0.24 & 0.79 & 0.08 \\
\hline Population density 1960 & 0.46 & 0.86 & 0.01 & 0.27 & 0.17 \\
\hline Malaria prevalence in 1960's & 0.34 & 0.02 & 0.78 & 0.00 & 0.14 \\
\hline Spanish colony & 0.11 & 0.01 & 0.25 & 0.01 & 0.01 \\
\hline Life expectancy in 1960 & 0.10 & 0.04 & 0.18 & 0.03 & 0.00 \\
\hline Fraction Confucian & 0.08 & 0.02 & 0.03 & 0.84 & 0.04 \\
\hline Latin American dummy & 0.08 & 0.00 & 0.05 & 0.82 & 0.04 \\
\hline African dummy & 0.07 & 0.03 & 0.00 & 0.81 & 0.04 \\
\hline Ethnolinguistic fractionalization & 0.07 & 0.10 & 0.03 & 0.04 & 0.00 \\
\hline Government consumption share 1960's & 0.05 & 0.02 & 0.08 & 0.09 & 0.00 \\
\hline Political rights & 0.05 & 0.09 & 0.00 & 0.00 & 0.00 \\
\hline Years open & 0.05 & 0.04 & 0.05 & 0.04 & 0.00 \\
\hline Fraction Muslim & 0.04 & 0.03 & 0.03 & 0.15 & 0.00 \\
\hline Fraction Buddhist & 0.04 & 0.04 & 0.00 & 0.28 & 0.00 \\
\hline Fraction GDP in mining & 0.04 & 0.03 & 0.02 & 0.15 & 0.00 \\
\hline Population density costal 1960's & 0.03 & 0.06 & 0.00 & 0.08 & 0.00 \\
\hline Higher education in 1960 & 0.03 & 0.03 & 0.03 & 0.00 & 0.00 \\
\hline (Imports+exports)/GDP & 0.03 & 0.03 & 0.02 & 0.02 & 0.00 \\
\hline Government share of GDP & 0.02 & 0.01 & 0.02 & 0.14 & 0.00 \\
\hline Fraction speaking foreign language & 0.02 & 0.02 & 0.02 & 0.03 & 0.00 \\
\hline Primary exports in 1970 & 0.02 & 0.00 & 0.04 & 0.00 & 0.00 \\
\hline Air distance to big cities & 0.02 & 0.04 & 0.00 & 0.00 & 0.00 \\
\hline Real exchange rate distortions & 0.02 & 0.02 & 0.01 & 0.02 & 0.00 \\
\hline Fraction population less than 15 & 0.02 & 0.03 & 0.01 & 0.00 & 0.00 \\
\hline Government consumption share d & 0.01 & 0.01 & 0.00 & 0.10 & 0.00 \\
\hline Fraction Protestant & 0.01 & 0.01 & 0.02 & 0.01 & 0.00 \\
\hline Fraction population in tropics & 0.01 & 0.01 & 0.01 & 0.03 & 0.00 \\
\hline Absolute latitude & 0.01 & 0.01 & 0.01 & 0.00 & 0.00 \\
\hline Civil liberties & 0.01 & 0.01 & 0.00 & 0.00 & 0.00 \\
\hline Colony dummy & 0.01 & 0.01 & 0.01 & 0.00 & 0.00 \\
\hline Revolution and coups & 0.01 & 0.01 & 0.01 & 0.00 & 0.00 \\
\hline Outward orientation & 0.01 & 0.01 & 0.00 & 0.00 & 0.00 \\
\hline Fraction Hindu & 0.01 & 0.01 & 0.00 & 0.00 & 0.00 \\
\hline Average inflation 1960-1990 & 0.01 & 0.01 & 0.00 & 0.00 & 0.00 \\
\hline European dummy & 0.00 & 0.00 & 0.00 & 0.01 & 0.00 \\
\hline Size of the economy & 0.00 & 0.01 & 0.00 & 0.00 & 0.00 \\
\hline Hydrocarbon deposits in 1993 & 0.00 & 0.01 & 0.00 & 0.00 & 0.00 \\
\hline Fertility in 1960 's & 0.00 & 0.01 & 0.00 & 0.00 & 0.00 \\
\hline Fraction population over 65 & 0.00 & 0.00 & 0.00 & 0.00 & 0.00 \\
\hline British colony & 0.00 & 0.01 & 0.00 & 0.00 & 0.00 \\
\hline English-speaking population & 0.00 & 0.00 & 0.01 & 0.00 & 0.00 \\
\hline Square of inflation $1960-1990$ & 0.00 & 0.01 & 0.00 & 0.00 & 0.00 \\
\hline Defense spending share & 0.00 & 0.01 & 0.00 & 0.00 & 0.00 \\
\hline Landlocked country dummy & 0.00 & 0.01 & 0.00 & 0.00 & 0.00 \\
\hline Religous intensity & 0.00 & 0.01 & 0.00 & 0.00 & 0.00 \\
\hline Oil-producing country dummy & 0.00 & 0.01 & 0.00 & 0.00 & 0.00 \\
\hline Time of independence & 0.00 & 0.01 & 0.00 & 0.00 & 0.00 \\
\hline Socialist dummy & 0.00 & 0.01 & 0.00 & 0.00 & 0.00 \\
\hline Fraction Catholic & 0.00 & 0.00 & 0.00 & 0.01 & 0.00 \\
\hline Population growth rate $1960-1990$ & 0.00 & 0.00 & 0.00 & 0.00 & 0.00 \\
\hline Nominal government GDP share 1960's & 0.00 & 0.01 & 0.00 & 0.00 & 0.00 \\
\hline Public education spending share in GDP in 1960's & 0.00 & 0.00 & 0.00 & 0.00 & 0.00 \\
\hline Capitalism & 0.00 & 0.01 & 0.00 & 0.00 & 0.00 \\
\hline Terms of trade growth in 1960 's & 0.00 & 0.00 & 0.00 & 0.00 & 0.00 \\
\hline Tropical climate zone & 0.00 & 0.00 & 0.00 & 0.00 & 0.00 \\
\hline Fraction spent in war $1960-1990$ & 0.00 & 0.00 & 0.00 & 0.00 & 0.00 \\
\hline War participation $1960-1990$ & 0.00 & 0.00 & 0.00 & 0.00 & 0.00 \\
\hline Land area & 0.00 & 0.00 & 0.00 & 0.00 & 0.00 \\
\hline Population in 1960 & 0.00 & 0.00 & 0.00 & 0.00 & 0.00 \\
\hline Fraction Orthodox & 0.00 & 0.00 & 0.00 & 0.00 & 0.00 \\
\hline Fraction of land area near navigable water & 0.00 & 0.00 & 0.00 & 0.00 & 0.00 \\
\hline Interior density & 0.00 & 0.00 & 0.00 & 0.00 & 0.00 \\
\hline Terms of trade ranking & 0.00 & 0.00 & 0.00 & 0.00 & 0.00 \\
\hline
\end{tabular}

